\title{
A novel method to correct for pitch and yaw patient setup errors in helical tomotherapy
}

\author{
Sarah A. Boswell ${ }^{\mathrm{a})}$ \\ Department of Medical Physics, University of Wisconsin, Madison, Wisconsin 53706 \\ Robert Jeraj \\ Department of Medical Physics, University of Wisconsin, Madison, Wisconsin 53706, and Jozef Stefan \\ Institute, Ljubljana, Slovenia \\ Kenneth J. Ruchala and Gustavo H. Olivera \\ TomoTherapy, Inc., Madison, Wisconsin 53717 \\ Hazim A. Jaradat \\ Department of Human Oncology, University of Wisconsin, Madison, Wisconsin 53706 \\ Joshua A. James and Alonso Gutierrez \\ Department of Medical Physics, University of Wisconsin, Madison, Wisconsin 53706 \\ Dave Pearson \\ TomoTherapy, Inc., Madison, Wisconsin 53717 \\ Gary Frank \\ Department of Medical Physics, University of Wisconsin, Madison, Wisconsin 53706 \\ T. Rock Mackie \\ Department of Medical Physics, University of Wisconsin, Madison, Wisconsin 53706, and TomoTherapy, \\ Inc., Madison, Wisconsin 53717
}

(Received 10 December 2004; revised 16 March 2005; accepted for publication 19 March 2005; published 20 May 2005)

\begin{abstract}
An accurate means of determining and correcting for daily patient setup errors is important to the cancer outcome in radiotherapy. While many tools have been developed to detect setup errors, difficulty may arise in accurately adjusting the patient to account for the rotational error components. A novel, automated method to correct for rotational patient setup errors in helical tomotherapy is proposed for a treatment couch that is restricted to motion along translational axes. In tomotherapy, only a narrow superior/inferior section of the target receives a dose at any instant, thus rotations in the sagittal and coronal planes may be approximately corrected for by very slow continuous couch motion in a direction perpendicular to the scanning direction. Results from proof-of-principle tests indicate that the method improves the accuracy of treatment delivery, especially for long and narrow targets. Rotational corrections about an axis perpendicular to the transverse plane continue to be implemented easily in tomotherapy by adjustment of the initial gantry angle. (C) 2005 American Association of Physicists in Medicine. [DOI: 10.1118/1.1914543]
\end{abstract}

\section{INTRODUCTION}

Pretreatment setup verification and a method to refine the patient position are requirements for every precision radiotherapy system. Helical tomotherapy employs a linear accelerator mounted on a CT gantry, thus allowing for CT verification of the daily target position. ${ }^{1,2}$ A coordinate system is shown in Fig. 1. The TomoTherapy HiArt machine automatically adjusts the pretreatment couch position to correct for setup errors along the $y$ and $z$ axes, and the $x$ position of the couch may be adjusted manually. Automatic roll correction (rotation about the $y$ axis) is accomplished by an adjustment to the initial gantry angle. However, errors in pitch (rotation about the $x$ axis) and yaw (rotation about the $z$ axis) must be corrected by physically rotating the patient about a prescribed point, an error-prone process that may necessitate further setup verification. For this reason, the HiArt software for registering the daily $\mathrm{CT}$ image with a planning $\mathrm{CT}$ image provides the option of ignoring pitch and yaw degrees of freedom when automatically determining the rigid-body setup parameters by a voxel-based registration algorithm. A visual check of the registered images ensures that the prescribed adjustments produce satisfactory results in all six degrees of freedom (i.e., ensures that any pitch or yaw setup errors are small enough to be negligible).

We seek an automated method to correct for small pitch and yaw setup errors in helical tomotherapy. ${ }^{3}$ This could be accomplished by using a treatment couch with rotational degrees of freedom, or by an adjustment to the MLC delivery pattern. ${ }^{4}$ In tomotherapy, because only a narrow $y$ extent of the tumor is in the beam at any instant, there also exists a unique set of options for implementing setup adjustments during treatment on a local basis. For example, in addition to the $y$ axis scanning motion, a couch could be designed with the capability of traveling at a very low velocity along the $z$ and $x$ axes during treatment to approximately correct for pitch and yaw setup errors, respectively. This method would 


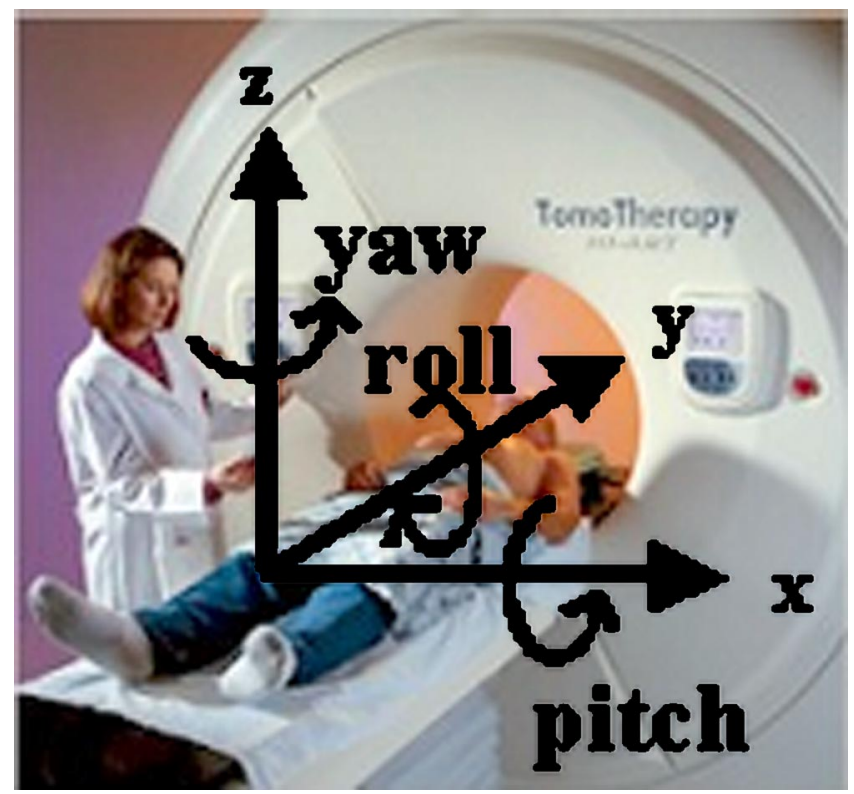

FIG. 1. Tomotherapy coordinate system with a patient in the head-first supine position. Positive rotations are clockwise, as viewed from the inferior end of the respective axis. Figure background courtesy of TomoTherapy, Inc.

also lend itself to the correction of $y$-dependent variations in pitch and yaw offsets, e.g., bending of a long target. Similarly, the local nature of tomotherapy delivery also presents the possibility of adjusting the couch speed relative to the gantry rotational rate to correct for $y$-dependent variations in roll, i.e., twisting of a long target. ${ }^{5}$

Use of motion along the $x$ axis to partially correct for a rigid-body yaw setup error is illustrated in Fig. 2 . The technique involves a corrective pretreatment translation to center the portion of the target that first enters the radiation field. Then during treatment, a very slow continuous motion is introduced along the $x$ axis to center the portion of the target that is currently in the beam. A mathematical description of the method follows.

Let the set of coordinates $(x, y)$ represent pixel locations within a patient who has been set up with an undesirable yaw rotation $-\theta$ about the $z$ axis with respect to the planned pixel coordinates $\left(x^{\prime}, y^{\prime}\right)$. To correct the daily patient setup, it will be necessary to rotate the patient by an angle $\theta$.

Rotation of a two-dimensional (2-D) vector $(x, y)$ by an angle $\theta$ about the $z$ axis (a yaw rotation) may be decomposed into shearing and scaling operations, as described by Snider and Mackie: ${ }^{6}$

$$
\begin{aligned}
{\left[\begin{array}{l}
x^{\prime} \\
y^{\prime}
\end{array}\right]=} & {\left[\begin{array}{cc}
\cos \theta & -\sin \theta \\
\sin \theta & \cos \theta
\end{array}\right]\left[\begin{array}{l}
x \\
y
\end{array}\right] } \\
= & {\left[\begin{array}{cc}
1 & -\tan \theta \\
0 & 1
\end{array}\right] } \\
& \times\left[\begin{array}{cc}
1 & 0 \\
\sin \theta \cos \theta & 1
\end{array}\right]\left[\begin{array}{cc}
\frac{1}{\cos \theta} & 0 \\
0 & 1
\end{array}\right]\left[\begin{array}{cc}
1 & 0 \\
0 & \cos \theta
\end{array}\right]\left[\begin{array}{l}
x \\
y
\end{array}\right] .
\end{aligned}
$$

From left to right, the terms on the right side of Eq. (1) represent the following: a shear along the $x$ axis, a shear along the $y$ axis, scaling along the $x$ axis, and scaling along the $y$ axis. Shearing may be defined as position-dependent translation, and shearing along the $x$ axis is accomplished in our application by introducing a secondary $x$ translation in conjunction with the $y$ axis couch travel. Shearing along the $y$ axis may not be implemented by a similar strategy because the couch scans along the $y$ axis. Scaling along the $x$ or $y$ axis would require the modification of delivery parameters in addition to couch travel.

Thus, Eq. (1) may be written as

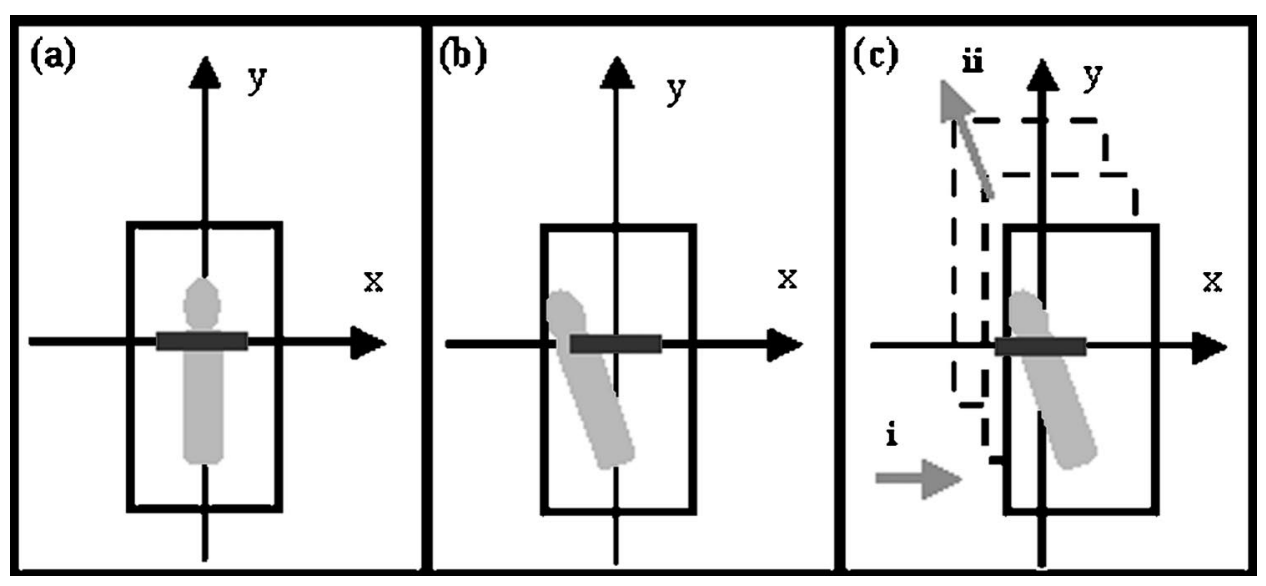

FIG. 2. Coronal view of the correction method. (a) A patient with a long and narrow tumor in the treatment position. The delivery isocenter is at the intersection of the $x$ and $y$ axes, and the radiation field is indicated by the small rectangle at that location. (b) Patient setup for daily treatment with a yaw setup error, the magnitude of which is exaggerated for illustration. (c) Prior to treatment, the couch was translated in the $x$ direction so that the first slice to be treated is $x$ centered, as indicated by arrow (i). Then, as the couch advances in the $y$ direction into the bore during treatment, a secondary motion in the $-x$ direction ensures that the current slice is always $x$ centered. The net direction of couch motion during treatment is indicated by arrow (ii). 


$$
\left[\begin{array}{l}
x \prime \\
y^{\prime}
\end{array}\right]=\mathbf{C}(\theta) \mathbf{N}(\theta)\left[\begin{array}{l}
x \\
y
\end{array}\right],
$$

where

$$
\begin{aligned}
& \mathbf{C}(\theta)=\left[\begin{array}{cc}
1 & -\tan \theta \\
0 & 1
\end{array}\right], \\
& \mathbf{N}(\theta)=\left[\begin{array}{cc}
1 & 0 \\
\sin \theta \cos \theta & 1
\end{array}\right]\left[\begin{array}{cc}
\frac{1}{\cos \theta} & 0 \\
0 & 1
\end{array}\right]\left[\begin{array}{cc}
1 & 0 \\
0 & \cos \theta
\end{array}\right] .
\end{aligned}
$$

We propose implementing the $\mathbf{C}(\theta)$ component of a yaw correction by couch travel along the $x$ axis. The $\mathbf{N}(\theta)$ com- ponent will be ignored because it is more difficult to implement. Thus, our proposed correction method reduces the delivery error due to a rotational setup error, but does not eliminate it.

By Eq. (1), the distance error $E(x, y, \theta)$ at each pixel in a patient due to an uncorrected rotational offset $-\theta$ is

$$
\begin{aligned}
& E(x, y, \theta) \\
& \quad=\sqrt{\left(x-x^{\prime}\right)^{2}+\left(y-y^{\prime}\right)^{2}} \\
& \quad=\sqrt{(x-(x \cos \theta-y \sin \theta))^{2}+(y-(x \sin \theta+y \cos \theta))^{2}} .
\end{aligned}
$$

Implementing the $\mathbf{C}(\theta)$ correction term reduces the error to

$$
R(x, y, \theta)=\sqrt{((x-y \tan \theta)-(x \cos \theta-y \sin \theta))^{2}+(y-(x \sin \theta+y \cos \theta))^{2}} .
$$

The residual error $R(x, y, \theta)$ is the result of ignoring $\mathbf{N}(\theta)$.

For simplicity, we have concentrated on an approximate correction of a yaw setup error. Compensation for a pitch setup error is completely analogous; simply replace $x$ with $z$ in the preceding discussion. The error due to an uncorrected pitch is independent of $x$, and the error due to an uncorrected yaw is independent of $z$.

The correction method is also expected to be useful for cases in which the registration software recommends pitch, yaw, and roll adjustments. $\mathbf{C}(\theta)$ corrections for pitch and yaw are implemented by couch motion along orthogonal axes, and thus would not interfere with each other. When the TomoTherapy registration software is used to determine translational offsets and rotational offsets about all three axes, the prescribed order of determination and implementation of adjustments is translations, pitch, yaw, roll. The HiArt tomotherapy machine provides automatic roll correction by adjusting the gantry start angle. Since roll is detected and corrected subsequent to any modifications to the patient position, there will be no problem with combining pitch and yaw corrections with a roll correction.

The magnitude of the translation required to center the first portion of the target that enters the beam [Fig. 2(c)] is $\sin \theta$ times the $y$ distance from the center of rotation to the superior end of the target. (For our purposes, the center of rotation is the point about which the registration software introduces rotations to register the daily image to the planning image. The designation of the rotation center does not affect the angle of the rotation correction, but compensating translational setup adjustments would be prescribed by the registration software if another center of rotation were chosen.) Equation (2) implies that the speed of motion along the secondary axis will be $\tan \theta$ times the scanning speed.

In practice, rotational setup errors to be corrected by this method will be on the order of five degrees or less. Rota- tional setup errors of greater magnitude should be corrected by repositioning the patient. Thus the prescribed speed along a secondary axis will always be less than $0.5 \mathrm{~mm} / \mathrm{s}$. The patient would not be expected to perceive such slow travel along a secondary axis, and there is plenty of room within the tomotherapy bore to accommodate the small travel distances needed to correct for rotational offsets of a few degrees.

It would also be feasible to use a similar correction method in axial tomotherapy. At each slice, the couch height and lateral position could be adjusted to correct for pitch and yaw setup errors. However, the method would not be as precise for axial tomotherapy because the corrective motion would be incremental, not continuous.

In terms of patient comfort and reliability, the proposed method represents a significant improvement over pushing the patient around on the couch to make fine rotational adjustments. The main drawback to our method is the residual error component $R(x, y, z)$. Alternative adjustment methods include daily replanning, modification of the delivery sinogram without replanning, and rotating the treatment couch. If fast enough algorithms became available, replanning based on the daily CT scans would provide better tumor coverage and normal tissue sparing than our proposed method. If not, the proposed on-line couch correction could be used in conjunction with off-line adaptive radiotherapy ${ }^{7}$ to further refine the dose distribution for subsequent fractions. On-line modification of the delivery sinogram without replanning, as proposed by Olivera, et al., ${ }^{4}$ would also be possible within the clinical time constraints. The delivery modification algorithm proposed by Olivera et al., could even be used in conjunction with the method proposed here to correct for the $y$-shear component of $\mathbf{N}(\theta)$ in Eq. (2).

Instead of modifying the tomotherapy couch design to allow motion along secondary axes during treatment, it 
would be possible to develop a tomotherapy couch with pitch and yaw degrees of freedom. (Tilting the tomotherapy gantry to account for pitch offsets would require significant modification to the system.) A group from the University of Michigan ${ }^{8,9}$ has developed a device that may be mounted on a treatment couch to make pitch and roll corrections to within $\pm 0.03^{\circ}$, with yaw corrections being accomplished by rotating the couch pedestal. The HexaPOD couch top by Medical Intelligence also provides six degrees of freedom in motion.

In comparison with existing rotating couch designs, the CT scanning geometry of helical tomotherapy would place additional design constraints on a five degrees of freedom couch (three translational degrees of freedom plus pitch and yaw, roll being accomplished by adjusting the tomotherapy gantry start angle). A five degrees of freedom tomotherapy couch would require more than a meter's range of motion along the $y$ axis. The tabletop must travel through the tomotherapy bore, but any additional couch supports must remain outside the bore. If the tomotherapy pedestal were not modified so that it could rotate to introduce yaw adjustments, the tabletop itself would need to rotate. And while our proposed method lends itself to the correction of $y$-dependent variations in pitch or yaw setup errors (bending of the patient anatomy), a five degrees of freedom couch would need to rotate during treatment delivery in order to do the same. Thus from an engineering standpoint, our proposed approach is simpler because the TomoTherapy couch already provides $x$ and $z$ control.

\section{MATERIALS AND METHODS}

While the clinical significance of an uncorrected or partially corrected pitch or yaw setup error depends on the patient anatomy and the treatment plan, the geometrical delivery error at each pixel location is governed simply by Eqs. (3) and (4). Regardless of the homogeneity of the treatment field or the target margins, each beamlet will deposit energy in the patient at a distance from the intended location that is a function of the magnitude of the rotation angle and the distance from the center of rotation. Results from calculations will be presented.

Experiments were also performed on the TomoTherapy machine to test the efficacy of the method in correcting yaw rotations during scan acquisition and treatment delivery. The proposed correction method requires a means of introducing couch motion along a secondary axis during treatment. As an initial test, we corrected for yaw rotational setup errors in a phantom using a small, motorized slider (Unislide Assy, Velmex, Inc., Bloomfield, NY; Slo-syn motor, Superior Electric, Danaher Motion, Rockford, IL; Panther LI driver, Intelligent Motor Systems, Inc., Marlborough, CT) placed on the treatment couch. The slider axis of motion was parallel to the $x$ axis (Fig. 3). The system is designed to move light objects with accurate speeds ranging from $0.8 \mu \mathrm{m} / \mathrm{s}$ to $0.33 \mathrm{~mm} / \mathrm{s}$. A particle board platform was placed across the slider, with the phantom on one end and a counterweight on the other end; thus the phantom was located at some distance from the

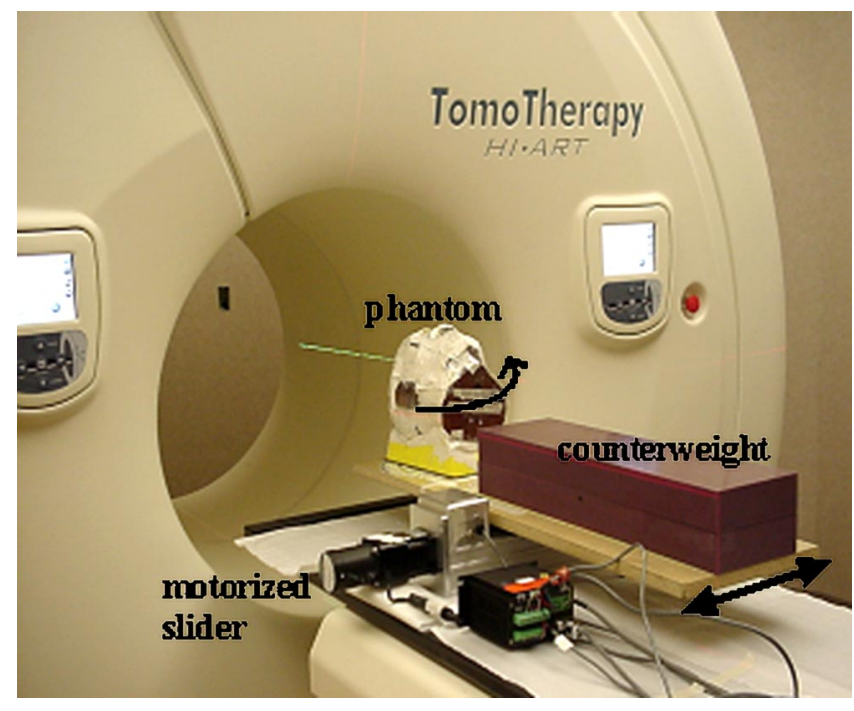

FIG. 3. Experimental setup. Yaw setup errors are manually introduced about the center of the head phantom as indicated by the curved arrow, and the motorized slider may be used to introduce a compensating motion to the platform on which the phantom rests. The slider moves in the positive or negative $x$ direction as indicated by the double-headed arrow.

metal slider so as not to introduce $\mathrm{CT}$ artifacts. The phantom can be manually rotated on a peg in the platform to introduce yaw rotations, which may be corrected for by motion along the $x$ axis.

The phantom was kVCT scanned with $1.25 \mathrm{~mm}$ slices. Then the apparatus was placed on the tomotherapy couch. We compared results for three different scenarios, corresponding to the cartoon panels in Fig. 2: (a) with the phantom setup in the planning position; (b) with an uncorrected yaw setup error (the magnitude of the error was determined by fusing an MVCT image to the planning image using the registration software), and (c) using the slider to introduce a shear along the $x$ axis to correct for the yaw setup error.

The setup of the phantom for the planning KVCT scan was such that the isocenter of the lasers in the CT room (about which the TomoTherapy registration software would later determine rotations) was located at the peg about which rotations are manually introduced. Ensuring that the actual center of rotation of the phantom corresponded to the software center of rotation conveniently prevented the registration software from reporting residual translational offsets when pure yaw rotations were introduced to the phantom about the peg.

\section{A. Compensating for yaw offsets during image acquisition}

Our first experiment takes advantage of the fact that the geometry of tomotherapy CT acquisition is identical to the geometry of tomotherapy treatment delivery. To qualitatively evaluate the effectiveness of the method, images of an anthropomorphic head phantom were acquired in each of the three scenarios just described. In clinical practice, the correction technique (scenario c) would not be applied during the acquisition of setup verification scans except perhaps for the 

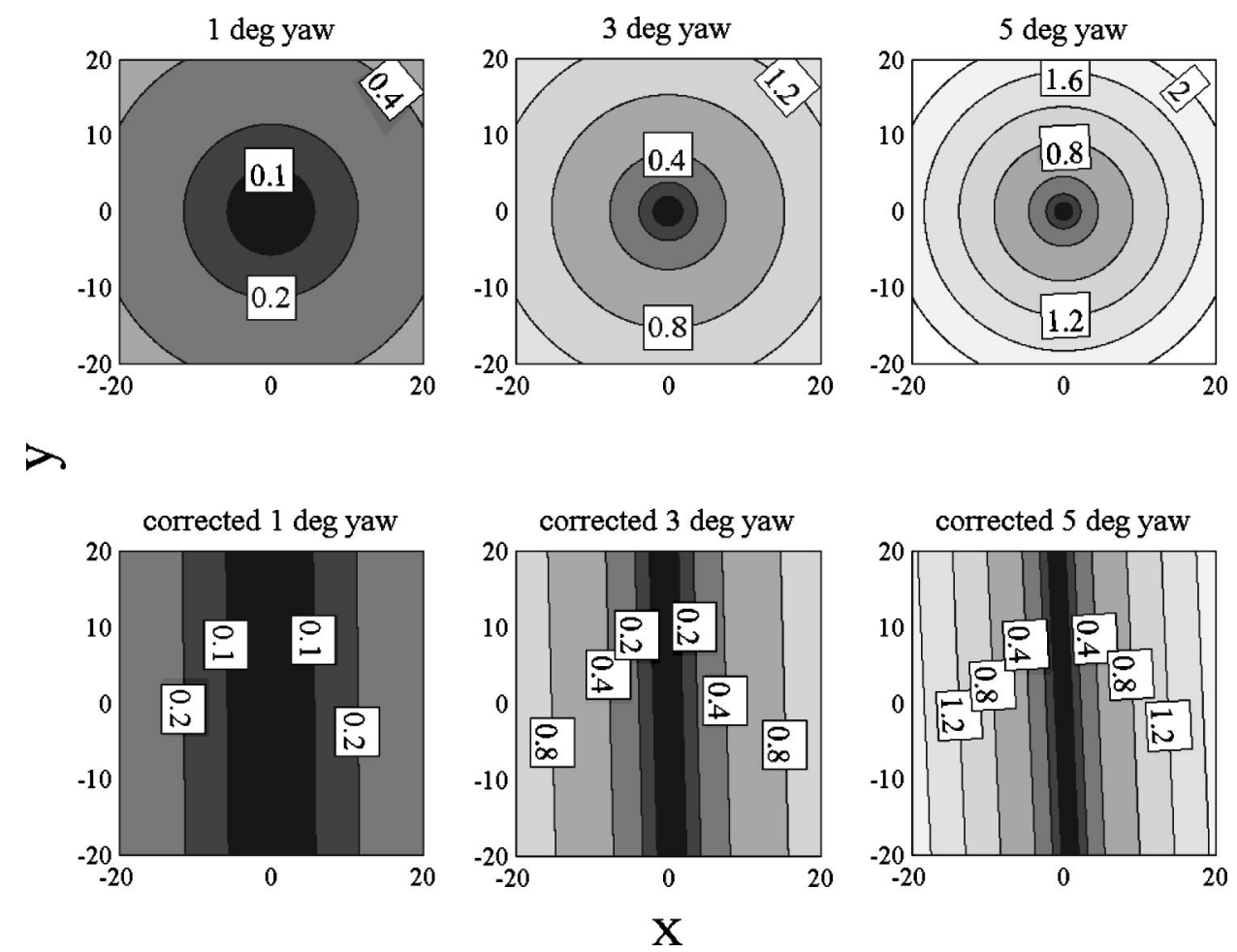

FIG. 4. Filled contours of Cartesian distance errors $E(x, y, \theta)$ due to uncorrected rotations of $1^{\circ}, 3^{\circ}$, and $5^{\circ}$ [Eq. (3)], and residual errors $R(x, y, \theta)$ after applying the correction technique [Eq. (4)]. Darker regions have smaller errors. The coordinate axes have the same units as the contour labels (e.g., $\mathrm{cm}$ ).

purpose of verifying the correction prior to treatment, but doing so provided images useful for visual evaluation of the correction method.

\section{B. Compensating for yaw offsets during delivery}

We also performed a quantitative experiment in the treatment mode using a phantom composed of two half-cylinders $(9 \mathrm{~cm}$ radius, $24 \mathrm{~cm}$ long). The phantom has acrylic walls and is filled with tap water. Kodak EDR2 film may be placed in the $x-y$ plane between the half-cylinders.

A CT scan of the phantom was acquired, contours were drawn, and the TomoTherapy optimizer was used to create a treatment plan with $95 \%$ of the target receiving $1.25 \mathrm{~Gy}$. The boundaries of the fairly uniform delivery pattern were designed to evaluate the accuracy of the correction method at various distances from the center of rotation along the $x$ and $y$ axes.

Next, the apparatus of Fig. 3 (the water phantom replacing the head phantom) was set up in the TomoTherapy treatment room according to the treatment plan, as verified by an MVCT scan. The treatment was delivered to expose a film in the $x-y$ plane between the halves of the phantom (scenario a). The film was removed, and the phantom was rotated on the peg attached to the platform. An MVCT scan of the phantom was acquired to determine the yaw offset, and a second film was exposed (scenario b). The corrective pretreatment translation was introduced, and a final film was exposed while the slider and motor were used to introduce corrective travel along the $x$ axis (scenario c).
The films were processed and scanned (resolution $0.0357 \mathrm{~cm}$ by $0.0357 \mathrm{~cm}$ ), and an in-house dosimetry program converted the pixel values to dose values based on an optical density curve determined by calibration films from the same film batch exposed previously. Changes in the film processor from the time of calibration to the time of this experiment were not accounted for, as absolute dosimetry was less important for this experiment than relative dose distributions of the three films exposed. To account for variations in film and machine output, the films were scaled so that the mean dose in the target was the same in each film. Pin marks identifying the phantom edges and laser locations, as well as the edge of the film itself (we used the Kodak film packaging that makes it possible to identify the edges of the film within the ReadyPack), were used to identify corresponding regions on the three films.

Distance-to-agreement and dose difference analysis were performed using in-house software. Dose difference maps are a good evaluation tool in low gradient regions, but tend to accentuate the error at the edges of the radiation field. Distance to agreement (DTA) is a better evaluation tool in high gradient regions. For each pixel in a film with an uncorrected or corrected yaw, the DTA is most commonly evaluated by searching the perfect setup image for the closest pixel with the same dose value, or for two pixels bracketing that dose value. ${ }^{10}$

An effective dose analysis measure should intelligently combine the dose difference and DTA analysis information. Two of the simplest approaches are to identify pixels that fail both dose difference and DTA tolerances, ${ }^{11}$ or to apply dose 
3 deg pitch, 3 deg yaw
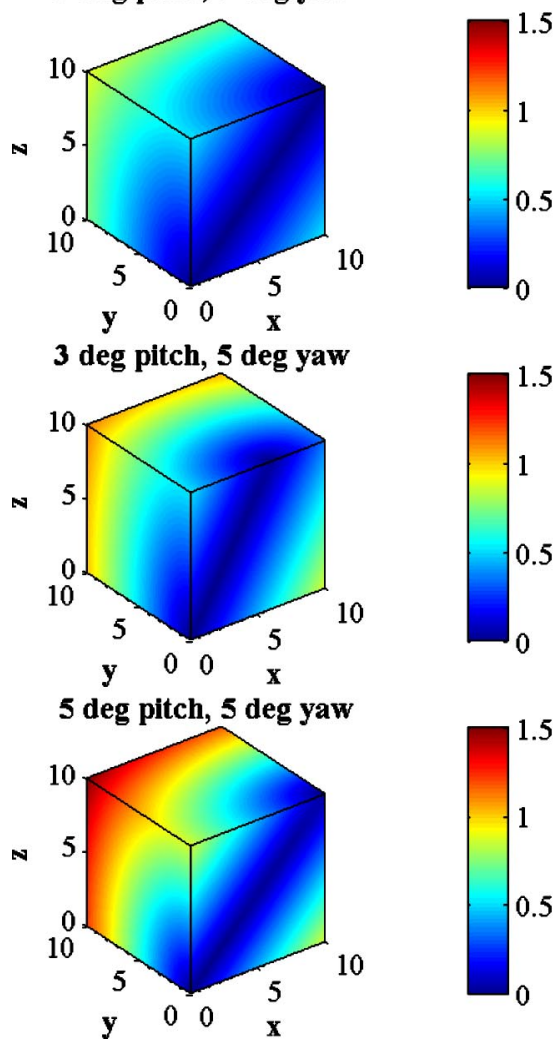

corrected 3 deg pitch, 3 deg yaw

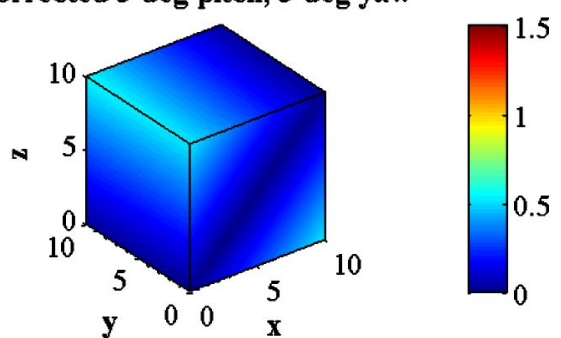

corrected 3 deg pitch, 5 deg yaw

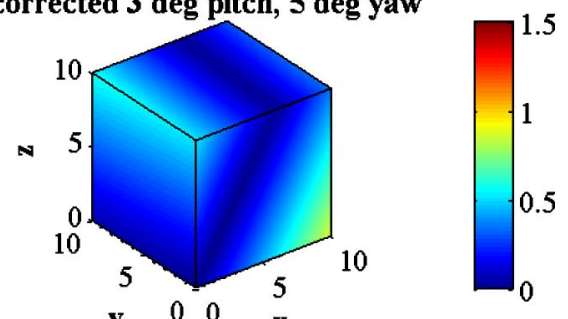

corrected 5 deg pitch, 5 deg yaw

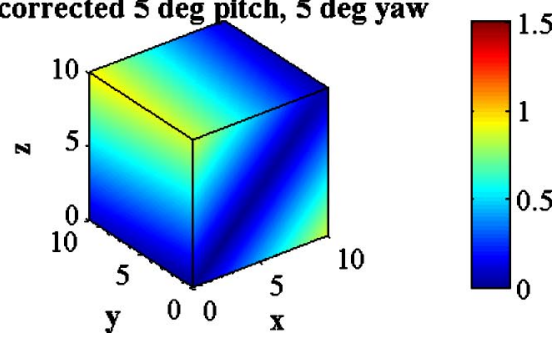

FIG. 5. 3-D Cartesian distance errors following uncorrected and corrected combined pitch and yaw setup errors. The coordinate axes have the same units as the error legend (e.g., cm). difference criteria to low gradient regions and DTA criteria to high gradient regions. ${ }^{12}$ The gradient was estimated at each pixel by finding the maximum slope between that pixel and one of its nearest neighbors.

\section{RESULTS}

\section{A. Theoretical calculations: Geometrical delivery error}

Figure 4 shows error contours in $\mathrm{cm}$ for uncorrected and $\mathbf{C}(\theta)$-corrected yaw setup errors of $1^{\circ}, 3^{\circ}$, and $5^{\circ}$. The error at each pixel is governed by Eq. (3) for an uncorrected yaw and Eq. (4) for a $\mathbf{C}(\theta)$-corrected yaw. The error due to an uncorrected or corrected yaw is independent of $z$. The error maps for the uncorrected cases are rings of concentric circles. At the rotation center $(0,0)$, there is no distance error, but the error increases with distance from the rotation center. In the corrected cases, the $y$ dependence is mostly removed by the $y$-dependent shear correction $\mathbf{C}(\theta)$, but the error increases with distance along the $x$ axis because we ignored the $x$-dependent shear term in $\mathbf{N}(\theta)$. Contours of constant error in the corrected cases are slightly tilted from the vertical because the $y$-scaling term in $\mathbf{N}(\theta)$ was also neglected. If the $x$ axis is renamed " $z$," then Fig. 4 represents errors for a pitch setup error instead of a yaw setup error.

A comparison of the uncorrected and corrected cases indicates that the correction method is expected to significantly reduce the error for tumor points with small $x$ coordinates but potentially large $y$ coordinates. Without the correction, the point $(x, y)=(2.5 \mathrm{~cm}, 20 \mathrm{~cm})$ would suffer a geometrical delivery error of $3.5 \mathrm{~mm}, 11 \mathrm{~mm}$, or $18 \mathrm{~mm}$, for $1^{\circ}, 3^{\circ}$, or $5^{\circ}$ rotations, respectively, whereas the couch correction reduces the errors to $0.5 \mathrm{~mm}, 2 \mathrm{~mm}$, or $3 \mathrm{~mm}$, respectively. Thus, for long and narrow tumors, e.g., spinal tumors, the correction technique is very effective. For short and narrow tumors, no correction would be needed because the distance errors due to a rotational offset would be small. For wide tumors, the couch correction would effectively reduce the error for points with small $x$ values, and would not further increase the error for points with large $x$ values.

Figure 5 shows 3-D error plots for an uncorrected pitch followed by an uncorrected yaw, and pitch and yaw simultaneously corrected by the method. The correction removes most of the $y$ dependence in the 3-D error cubes.

\section{B. Compensating for yaw offsets during image acquisition}

Megavoltage images were acquired in the planning position (scenario a), with an uncorrected yaw offset (scenario b), and during compensating couch translation (scenario c). The MVCT images were then registered to a planning $\mathrm{kVCT}$ image. The experiment was performed four times, with offsets ranging from $0.7^{\circ}$ to $5.6^{\circ}$. The theoretical limits for accuracy of the fusion software are governed by the scan resolution (accuracy to within $1 / 2$ pixel and $1 / 2 \mathrm{deg})^{2}$ Registration results indicate that the compensating couch translation successfully corrected for the yaw offsets to within $0.5^{\circ}$. 

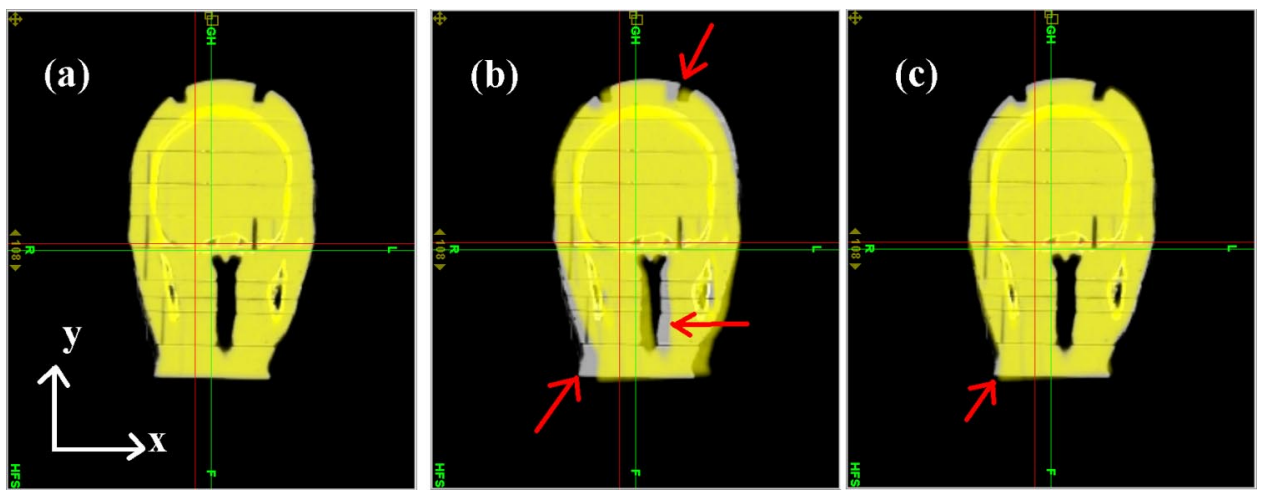

FIG. 6. Results from the qualitative experiment to test the correction method. Figure labels correspond to Fig. 2 frames (a), (b), and (c). (a) Coronal view of a planning kVCT image of the Rando head phantom fused to an MVCT image in the planning position with no rotational offsets. (b) A 5.6 rotation was introduced to the phantom about a point indicated by the green laser crossing (the lower right pair of lasers), and the rotational offset is visible when an MVCT image is acquired and overlaid on the kVCT image, especially in the regions indicated by the arrows. (c) A compensating motion in $x$ was introduced to the phantom as a final MVCT scan was being acquired, and the rotational offset is no longer visible, although there is a slight warping of the MVCT image far from the $x$ center due to the nature of the approximation, as most clearly visible in the corner indicated by the arrow.

Translational differences between the registration results of the perfect setup (scenario a) and the corrected setup (scenario c) were also well within the $1 / 2$ pixel limit.

Results for the $5.6^{\circ}$ case are shown in Fig. 6. It is evident that the corrective motion improves $\mathrm{CT}$ registration with the planning image, although the shape of the corrected image is sheared. The effects of this shearing are more severe at large distances along the $x$ axis from the center of rotation.

\section{Compensating for yaw offsets during treatment delivery}

Films were exposed when the cylindrical water phantom was in the planning position, with uncorrected yaw rotations to the phantom of $3.4^{\circ}$ and $6.4^{\circ}$, and with compensating couch translation for the two cases.

Figure 7 shows film dose distributions for uncorrected and
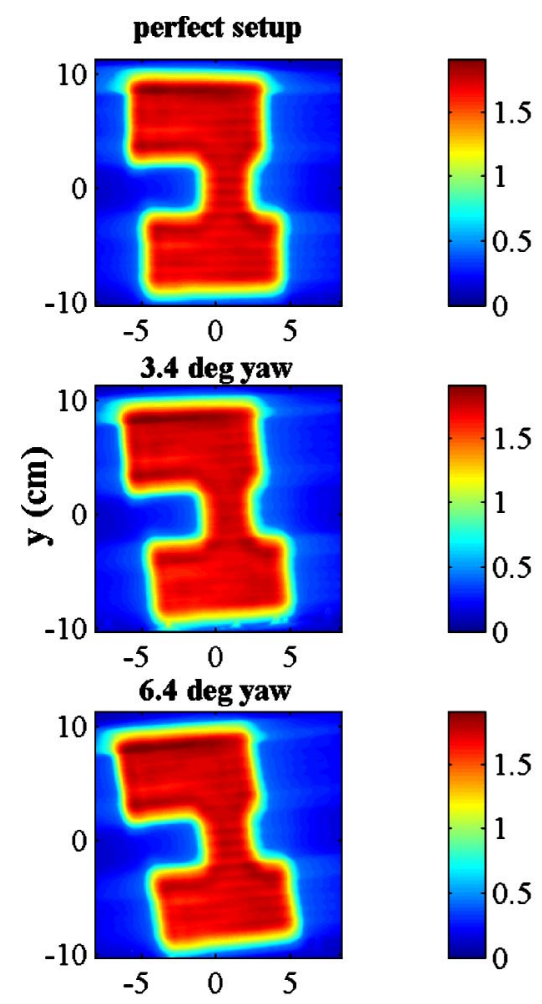
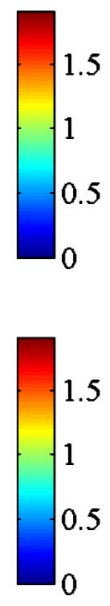
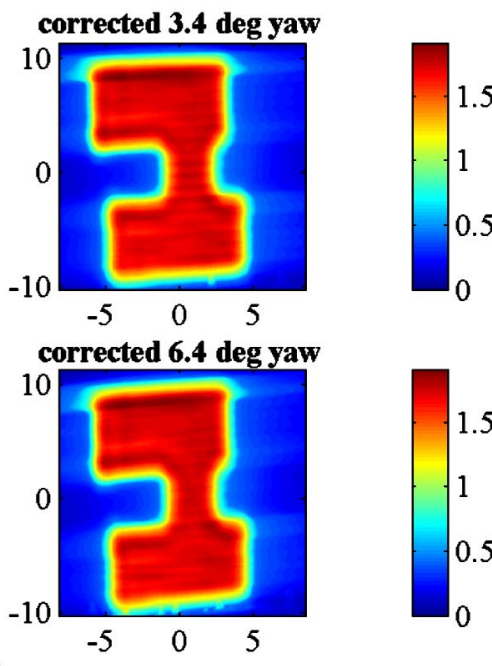

FIG. 7. Film dose distributions (Gy) from the quantitative test of the correction method. Film was placed in the coronal plane between halves of a cylindrical water phantom. The field is about $20 \mathrm{~cm}$ long, with field edges at $1,2,3,4,5$, and $6 \mathrm{~cm}$ along the $x$ axis from the center of yaw rotation $(0,0)$. $95 \%$ of the target volume received $1.25 \mathrm{~Gy}$. The axes of the distribution are in $\mathrm{cm}$. A scaling factor was introduced to ensure that the mean dose in all films was the same. 

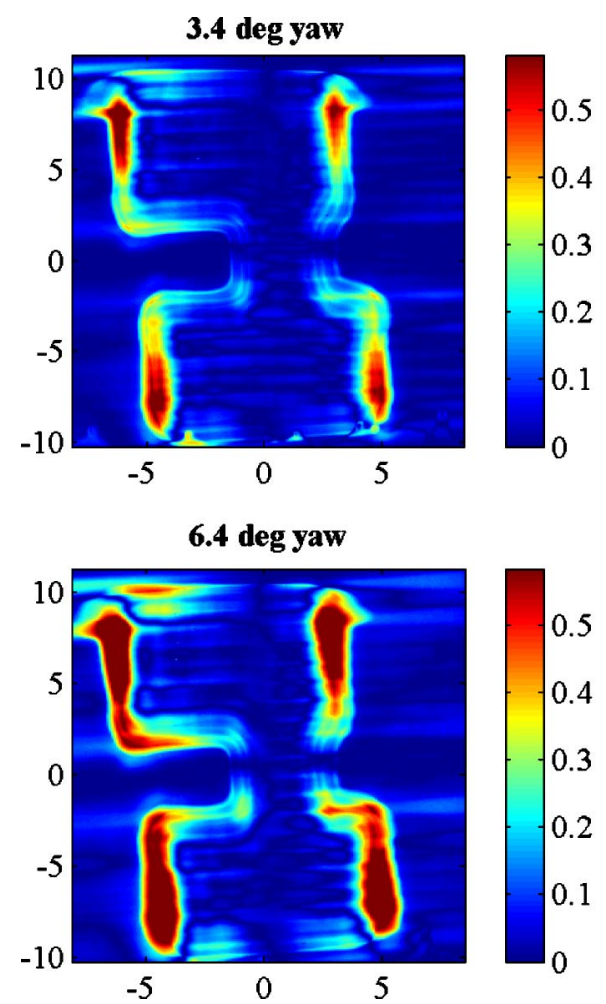
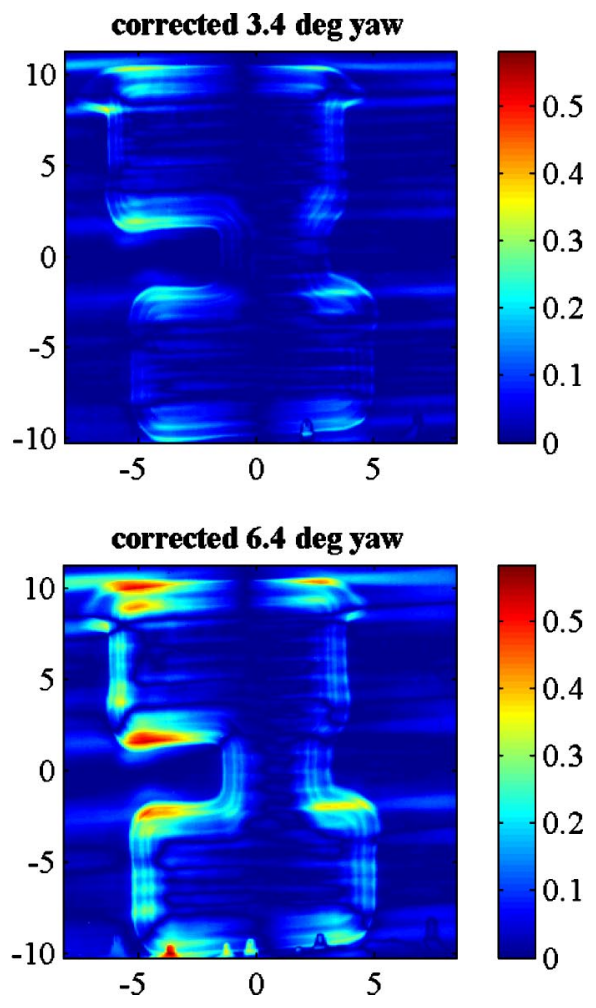

FIG. 8. Differences [Gy] between the perfect setup film and dose distributions shown in Fig. 7. The axes of the distribution are in $\mathrm{cm}$. It is instructive to consider how the dose differences increase with distance from the center of rotation $(0,0)$ along each axis. The horizontal stripes in the dose difference maps are due to interplay of the helical delivery pattern with the beam divergence, as described by Kissick et al. (Ref. 13). corrected yaw rotations of $3.4^{\circ}$ and $6.4^{\circ}$. Field edges that were formerly parallel to the $x$ or $y$ axis become slanted as a result of an uncorrected rotation. The correction straightens out the field edges that are supposed to be parallel to the $y$ axis, without affecting the angle of the field edges that are supposed to be parallel to the $x$ axis.

Dose differences between the perfect setup and the uncorrected and corrected yaw cases are shown in Fig. 8. Since the delivery field is fairly homogeneous, dose differences are largest at the edges of the field (i.e., where DTA analysis provides more useful results). Examining the variation in dose differences along the $x$ and $y$ field boundaries in the uncorrected case shows that the error increases with the $y$ distance from the center of rotation. The error also increases with the $x$ distance from the center of rotation, although the effect is less prominent since the treatment field is longer than it is wide. In the corrected case, the overall error is lower, and most of the $y$ dependence in the error has been removed, although the error dependence with $x$ is still noticeable. Film processor artifacts are visible on the $-y$ end of some films. The horizontal stripes in the dose difference maps are due to interplay of the helical delivery pattern with the beam divergence, as described by Kissick et al. ${ }^{13}$ and Mackie et al. ${ }^{14}$

Figure 9 shows DTA maps in regions where the local dose gradient is greater than $30 \%$ of the maximum dose per $\mathrm{cm}$. (The maximum dose in the film with the perfect setup was 1.91 Gy.) The first and second moments of these DTA distributions are also listed. The mean DTA (first moment) is reduced by the correction method in both cases.

The first two columns of Table I show the percentage of pixels that pass either the DTA or the dose criterion of $3 \mathrm{~mm}$ or $3 \%$ or $5 \mathrm{~mm}$ or $5 \%$. For purposes of beam commissioning, Van Dyk et al. ${ }^{12}$ have proposed tolerances of $4 \mathrm{~mm}$ DTA in high gradient regions (defined by Van Dyk as greater than $30 \%$ of the maximum dose per $\mathrm{cm}$ ), and $3 \%$ of the normalization dose in low gradient regions. These results are shown in the middle column of Table I. For patient specific quality assurance, the tolerances would have to be increased as compared to those for beam commissioning. The last column of Table I shows the percentage of pixels within $5 \mathrm{~mm}$ DTA in high gradient regions (defined here as regions where the gradient in both films is greater than $20 \%$ of the maximum dose per $\mathrm{cm}$ in the perfect setup film), and the percentage of pixels with differences of no more than $5 \%$ of the maximum dose in low gradient regions.

Regardless of the criteria employed, the distributions obtained during corrective $x$ motion of the couch have more passing pixels than the uncorrected distributions. As compared to the uncorrected cases, the left column of Table I shows that about $14 \%$ more of the total pixels in the corrected cases agree with the perfect setup film to within $3 \%$ of the maximum dose or $3 \mathrm{~mm}$ DTA. The middle and right columns of Table I show that the correction method increases the percentage of pixels in high gradient regions that pass the specified DTA criteria by $16 \%$ or $6 \%$ for a $3.4^{\circ}$ yaw, and by $28 \%$ or $23 \%$ for a $6.4^{\circ}$ yaw. The correction method increases the percentage of pixels in low gradient regions that pass the specified dose criteria by $10 \%$ or $7 \%$ for a $3.4^{\circ}$ yaw, and by $10 \%$ or $11 \%$ for a $6.4^{\circ}$ yaw. If the treatment field were less homogeneous, the increase in passing pixels due to the correction would likely be more dramatic. As expected, the cor- 

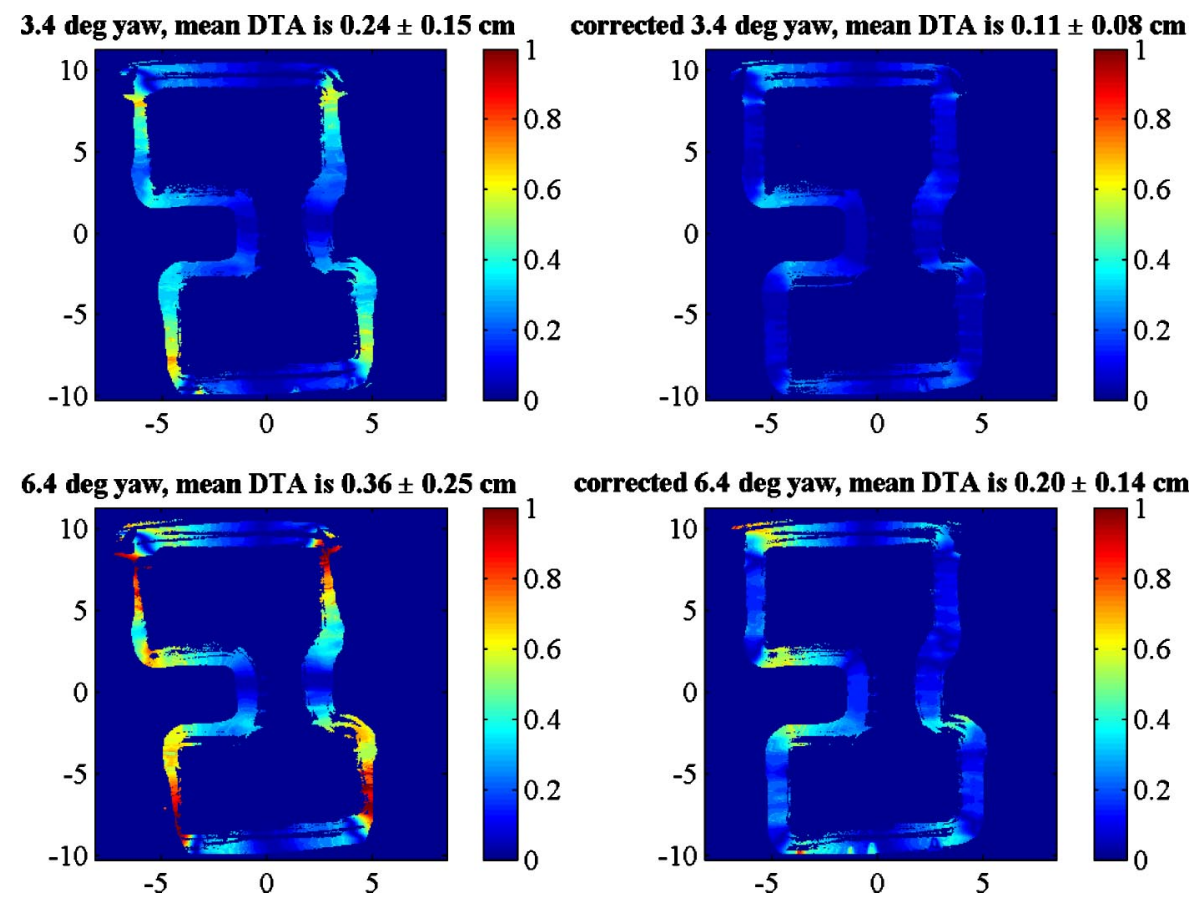

FIG. 9. Distance to agreement $(\mathrm{cm})$ between the perfect distribution and uncorrected and corrected distributions where the local gradient is greater than $30 \%$ of the maximum dose per $\mathrm{cm}$. rection method does not remove all of the error, and the corrected $6.4^{\circ}$ case has fewer passing pixels than the corrected $3.4^{\circ}$ case.

\section{DISCUSSION AND CONCLUSIONS}

Patient treatments in helical tomotherapy could be improved by an automated method to correct for any pitch and yaw rotational setup errors detected by registration of a pretreatment MVCT image to a planning image. The shear component $\mathbf{C}(\theta)$ of a pitch or yaw correction [Eq. (2)] may be implemented automatically by introducing a low-velocity motion along the $z$ or $x$ axis, respectively. If the compensating couch motion is applied during delivery to a $5 \mathrm{~cm}$ by
$5 \mathrm{~cm}$ by $40 \mathrm{~cm}$ treatment region (extends $\pm 2.5 \mathrm{~cm}$ along the $x$ and $z$ axes and $\pm 20 \mathrm{~cm}$ along the $y$ axis from the center of rotation), the maximum residual delivery error at any point in the region has been calculated to be less than $3 \mathrm{~mm}$ for a $5^{\circ}$ pitch or yaw, or $4 \mathrm{~mm}$ for a $5^{\circ}$ pitch and a $5^{\circ}$ yaw. Because the residual error after applying the correction method increases with the rotation angle, it should only be used for small refinements to the patient position, and should not become a substitute for repositioning to correct for large rotational setup errors, e.g., angles greater than $5^{\circ}$.

The method has been stated to work best at small $x$ and $z$ distances from the center of rotation because the $x$ - and $z$-dependent shear correction components are ignored by the

TABLE I. Percentage of passing pixels in uncorrected and corrected films using various evaluation criteria. Each film is compared to the perfect setup film. Dose differences between the two films are expressed as a percentage of the maximum dose in the perfect setup film. For a pair of corresponding pixels in the two images to be considered to be in the high gradient region, the local gradient in each image must be greater than the specified percentage of the maximum dose in the perfect setup film per $\mathrm{cm}$.

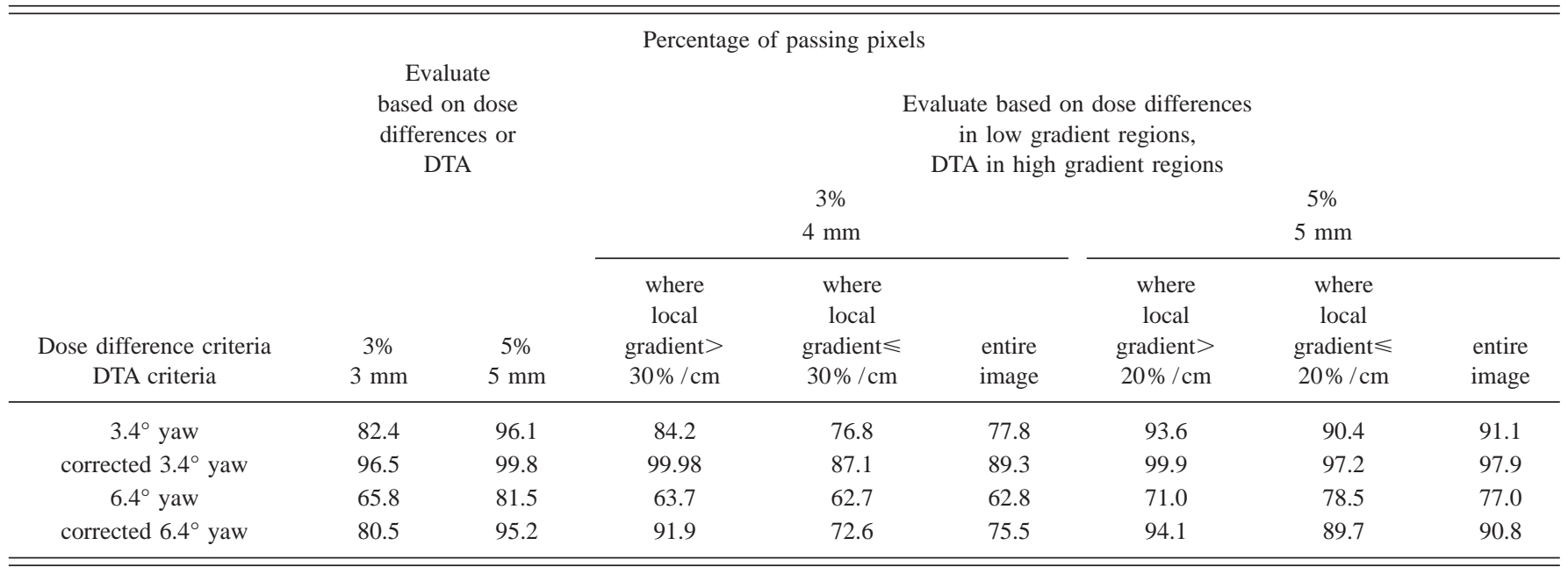


partial correction, and the error due to ignoring these components increases with $x$ and $z$ distance from the center of rotation. However, the designation of the center of rotation is an artificial construction of the registration software, thus the method also works for narrow targets which are not centered in $x$ and $z$. Given a set of adjustments recommended by the rotation software that includes rotations about a specified point, it would be easy to implement appropriate translations that effectively reassign the $x$ and $z$ center of rotation coordinates to the center of the target. If the software recommends translations $\left(C_{x}, C_{y}, C_{z}\right)$ followed by a pitch rotation $P$ and a yaw rotation $Y$ about $(0,0,0)$, we could effect the same patient setup by first translating by $\left(C_{x}+T_{x}, C_{y}+T_{y}, C_{z}+T_{z}\right)$, where the additional translations are

$$
\begin{aligned}
& T_{x}=R_{x}(1-\cos Y)-R_{y} \sin Y, \\
& T_{y}=R_{x} \sin Y \cos P+R_{y}(1-\cos Y \cos P)-R_{z} \sin P, \\
& T_{z}=-R_{x} \sin Y \sin P+R_{y} \sin P \cos Y+R_{z}(1-\cos P) .
\end{aligned}
$$

We could then follow the steps to partially correct for rotations $P$ and $Y$ about the center of the target $\left(R_{x}, R_{y}, R_{z}\right)$, where $\left(R_{x}, R_{y}, R_{z}\right)$ is defined for the target in the planned setup position. However, a yaw or pitch in a target consisting of two narrow structures separated by a large $x$ or $z$ distance would not be appropriately corrected by the compensating couch motion because it is effectively a "wide" target.

The proposed correction method ultimately requires a new tomotherapy couch that can travel slowly along the $x$ and $z$ axes during treatment. It should be possible to redesign the couch to move along the secondary axes at speeds of less than $0.5 \mathrm{~mm} / \mathrm{s}$ with an accuracy similar to that of the motorized slider used for the initial experiments. Tests of the method to correct for yaw setup errors in a phantom indicate that the correction method improves the percentage of points passing DTA and dose difference criteria by several percent. In practice, the magnitude of the improvement will depend on the characteristics of the treatment field, and the efficacy of the method to correct for rotations of typical magnitudes in clinical tomotherapy treatment plans is the subject of a future investigation. The method is also expected to work when both pitch and yaw rotational offsets are present.

\section{ACKNOWLEDGMENTS}

This work was partially supported by a grant from the NIH and by TomoTherapy, Inc. Thanks to Dr. Harry Keller,
Dr. Michael Kissick, Ryan Flynn, Dr. Wolfgang Tomé, Dr. John Fenwick, Brad Bennett, Dr. Susanta Hui, Stewart Becker, Oliver Langner, Jihad Al Sadah, Wayne Thatcher, Dr. Jacques Mallah, and Emilee Soisson for suggestions and technical assistance.

${ }^{\text {a)} E l e c t r o n i c ~ m a i l: ~ s a b o s w e l l @ w i s c . e d u ~}$

${ }^{1}$ G. H. Olivera, D. M. Shepard, K. J. Ruchala, J. S. Aldridge, J. Kapatoes, E. E. Fitchard, P. J. Reckwerdt, G. Fang, J. Zachman, and T. R. Mackie, "Tomotherapy," in The Modern Technology of Radiation Oncology, edited by J. Van Dyk (Medical Physics Publishing, Madison, 1999).

${ }^{2}$ K. J. Ruchala, G. H. Olivera, and J. Kapatoes, "Limited-data image registration for radiotherapy positioning and verification," Int. J. Radiat. Oncol., Biol., Phys. 54, 592-605 (2002).

${ }^{3}$ S. Boswell, R. Jeraj, A. Gutierrez, G. H. Olivera, H. A. Jaradat, D. Pearson, G. Frank, K. J. Ruchala, M. W. Kissick, H. Keller, B. Bennett, S. Hui, S. J. Becker, O. Langner, and T. R. Mackie, "A novel method to correct for rotational patient setup errors in helical tomotherapy," Med. Phys. 31, 1779 (2004).

${ }^{4}$ G. H. Olivera, E. E. Fitchard, P. J. Reckwerdt, K. J. Ruchala, and T. R. Mackie, "Modifying a plan delivery without re-optimization to account for patient offset in tomotherapy," Proceedings of the IEEE 22nd Annual International Conference, Engineering in Medicine and Biology Society, 2000, 1, pp. 441-444.

${ }^{5}$ J. D. Fenwick, W. A. Tomé, H. A. Jaradat, S. K. Hui, J. A. James, J. P. Balog, C. N. DeSouza, D. B. Lucas, G. H. Olivera, T. R. Mackie, and B. R. Paliwal, "Quality assurance of a helical tomotherapy machine." Phys. Med. Biol. 49, 2933-2953 (2004).

${ }^{6}$ A. Snider and T. R. Mackie, "Continuous antialiased 2-D and 3-D transformations in discrete space," Comput. Phys. 4, 78-81 (1990).

${ }^{7}$ D. Yan, F. Vicini, J. Wong, and A. Martinez, "Adaptive Radiation Therapy," Phys. Med. Biol. 42, 123-132 (1997).

${ }^{8}$ D. C. Hornick, D. W. Litzenberg, K. L. Lam, J. M. Balter, J. Hetrick, and R. K. Ten Haken, "A tilt and roll device for automated correction of rotational setup errors." Med. Phys. 25, 1739-1740 (1998).

${ }^{9}$ D. W. Litzenberg, J. M. Balter, D. C. Hornick, K. L. Lam, and R. K. Ten Haken, "A mathematical model for correcting patient setup errors using a tilt and roll device." Med. Phys. 26, 2586-2588 (1999).

${ }^{10}$ D. A. Low, W. B. Harms, S. Mutic, and J. A. Purdy, "A technique for the quantitative evaluation of dose distributions," Med. Phys. 5, 656-661 (1998).

${ }^{11}$ A. Cheng, W. B. Harms, R. L. Gerber, and J. W. Wong, "Systematic verification of a three-dimensional electron beam dose calculation algorithm," Med. Phys. 23, 685-693 (1996).

${ }^{12}$ J. Van Dyk, R. B. Barnett, J. E. Cygler, and P. C. Shragge, "Commissioning and quality assurance of treatment planning systems," Int. J. Radiat. Oncol., Biol., Phys. 26, 261-273 (1993).

${ }^{13}$ M. W. Kissick, J. D. Fenwick, J. A. James, R. Jeraj, J. M. Kapatoes, H. Keller, T. R. Mackie, G. H. Olivera, and E. T. Soisson, "The helical tomotherapy thread effect," Med. Phys. 32, 1414 (2005).

${ }^{14}$ T. R. Mackie, G. H. Olivera, J. M. Kapatoes, K. J. Ruchala, J. P. Balog, W. A. Tomé, S. Hui, M. W. Kissick, Chuan Wu, R. Jeraj, P. J. Reckwerdt, P. Harari, M. Ritter, L. Forrest, J. S. Welsh, and M. P. Mehta, "Helical tomotherapy," Intensity Modulation Radiation Therapy: The State of the Art (Medical Physics Publishing, Madison, WI, 2003), pp. 247-284. 